\title{
A note on field redefinitions and higher-spin equations
}

\author{
Massimo Taronna $a^{1, \star, \star \star}$ \\ ${ }^{1}$ Physique Théorique et Mathématique \\ Université Libre de Bruxelles and International Solvay Institutes \\ ULB-Campus Plaine CP231, 1050 Brussels, Belgium
}

\begin{abstract}
In this note we provide some details on the quasi-local field redefinitions which map interactions extracted from Vasiliev's equations to those obtained via holographic reconstruction. Without loss of generality, we focus on the source to the Fronsdal equations induced by $s-0-0$ couplings.
\end{abstract}

\section{Introduction}

The problem of admissible functional-classes has been of recent interest in the context of higher-spin (HS) theories [1]. In particular, in [2, 3] the quadratic interaction term sourcing Fronsdal's equations was extracted from Vasiliev's equations obtaining a pseudo-local expression of the schematic form: ${ }^{1}$

$$
\square \phi_{\mu(s)}+\ldots=\sum_{l=0}^{\infty} \frac{j_{l}}{l ! l !} \square^{l}(\nabla \ldots \nabla \phi \nabla \ldots \nabla \phi)_{\mu(s)},
$$

with coefficients $j_{l} \sim \frac{1}{l^{3}}$ for $l \rightarrow \infty$ for any choice of the spin $s$. The asymptotic behaviour of the coefficients raised the important question whether the backreaction extracted is or not strongly coupled $^{2}$. Furthermore, a key question whose study was undertaken in $[5,6]$ was whether it is possible to extract the coefficients of the canonical Metsaev vertices with finitely many derivatives from the above tails. Indeed most of the coefficients at the cubic order are unphysical, since they can be removed by local field redefinitions. In this respect, the full list of Metsaev-like couplings was indeed extracted holographically in [7] and amounts to a finite number of coefficients for any triple of spins, to be contrasted to the above infinite set (see also $[8,9]$ for the analogous string theory computation and corresponding cubic couplings). ${ }^{3}$

\footnotetext{
^e-mail: massimo.taronna@ulb.ac.be

$\star \star$ Postdoctoral Researcher of the Fund for Scientific Research-FNRS Belgium.

${ }^{1}$ Notice that in the following we use the schematic notation $\square^{l} \sim \ldots \nabla_{\mu(l)} \phi \ldots \nabla^{\mu(l)} \phi$. We give precise formulas for the above contractions in the spinor language in the following section in eq. (38). In this section all formulas are schematic and provide some intuition on their generic structure.

${ }^{2}$ Preliminary questions of this type were raised in [4].

${ }^{3}$ It is important to stress that in a fully non-linear HS theory it is expected that the appropriate field frame which makes HS geometry manifest will entail all of the above coefficients. The situation should be similar to the Einstein-Hilbert cubic couplings which are dressed by improvement terms that can be removed by a field redefinition at the cubic order. This is a further key motivation to understand these higher-derivative tails.
} 
Remarkably, the pseudo-local nature of the above currents implies that the only way to relate them to their Metsaev-like counterparts is via a pseudo-local field redefinition of the same schematic form:

$$
\phi_{\mu(s)} \rightarrow \phi_{\mu(s)}^{\prime}=\phi_{\mu(s)}+\sum_{l=0}^{\infty} \frac{a_{l}}{l ! l !} \square^{l}(\nabla \ldots \nabla \phi \nabla \ldots \nabla \phi)_{\mu(s)} .
$$

This result has motivated a renewed interest in the analysis of the admissible functional classes in HS theories. Indeed, an arbitrary pseudo-local redefinition is sufficient to remove all pseudo-local current interactions $[2,10]$. A proposal based on the invariance of the holographic Witten-diagrams was put forward in [5, 6], while in [11] (see [12] for further details) it was proposed to study classes of functions in $z$ and $y$ oscillators which are closed under star product multiplication. The aim of this note is to elaborate on these results from various perspectives, and to present the explicit form of the field redefinitions mapping the pseudo-local back-reaction (1) to its canonical (local) form.

In the following we list/summarise some relevant points of this analysis, together with the main results of this note, leaving the details of the derivation to the following sections:

- Defining the canonical $s$-derivative current made out of two scalars as:

$$
J_{\mu(s)}^{\mathrm{can}}=i^{s} \phi \stackrel{\leftrightarrow}{\nabla}_{\mu(s)} \phi,
$$

the redefinition which allows to bring the pseudo-local backreaction (1) to its canonical form:

$$
\underbrace{\square \phi_{\mu(s)}+\ldots}_{\mathcal{F}_{\mu(s)}}=\alpha_{s} J_{\mu(s)}^{\mathrm{can}},
$$

has the structure (2) with the following choice of coefficients:

$$
a_{l-1}=\frac{2 l+s+2}{2}\left(\frac{l !}{(l+s+1) !}\right)^{2}\left[p_{s}(l)+\# \alpha_{s}\right] .
$$

Above $p_{s}(l)$ a polynomial of degree $2(s-1)$ in $l$ while we have left the coefficients $\alpha_{s}$ arbitrary. In this way it is transparent to compare redefinitions that give different answers for the overall coefficient of the canonical current in (4). Moreover, only one of the redefinitions considered above gives the coupling constant which matches the one derived in [7, 13]. Keeping track of field normalisations (see Appendix B), in the type A theory the choice expected from holography is:

$$
\alpha_{s}=2^{1-s} g^{2} N_{0}^{2},
$$

which has a simple spin-dependence up to a spin-independent factor proportional to the normalisation of the scalar field kinetic term and to the HS coupling constant $g$.

- The value (6) for the coupling constant can be fixed using Noether procedure to the quartic-order (see e.g. [2] for the $3 \mathrm{~d}$ computation using admissibility condition) and was reconstructed from Holography in $[7,13]$. So far, however, it was not possible to fix all cubic couplings using only the Noether procedure. That this should be possible in principle is suggested by the result of Metsaev in the light-cone gauge $[14,15] .{ }^{4}$ Furthermore, the implications of the field redefinition mapping the theory to its canonical form at cubic order should be analysed at the quartic order. Such non-local redefinitions would generate a non-local quartic coupling. To appreciate the issue it might be worth

\footnotetext{
${ }^{4}$ In covariant language cubic couplings, including highest derivative ones, should be fixed by global part of the HS symmetry from the equation $\delta^{(1)} S^{(3)} \approx 0$.
} 
noting that the above redefinitions can generate quartic couplings which differ from each other by single-trace blocks in the corresponding conformal block expansion (see e.g. $[6,13,16,17]$ ). It is also conceivable that, at the quartic order another non-local redefinition will be needed to compensate the cubic redefinition and the additional non-local tails which would arise. The problem of finding a non-perturbative redefinition which relates the above tails to standard HS equations is so far open. In this note we restrict the attention to the lowest non-trivial order.

- The leading asymptotic behaviour for $l \rightarrow \infty$ of the coefficients in the field redefinition (5) is spinindependent and equal to $\frac{1}{l^{3}}$. Therefore, all the redefinitions considered above belong to the same functional space as the current in eq. (1) itself. This seems to suggest that the "admissible" functional class for field-redefinitions may be smaller than the corresponding "admissible" functional space for interactions.

- Notice that choosing a different coupling constant $\alpha_{s}$ for the canonical current amounts to a subleading contribution in (5) with spin-dependent behaviour $\sim \frac{1}{l^{2 s+1}}$ for the coefficients $a_{l}$. Changing the overall coefficient $\alpha_{s}$ of the current in (4) by $\epsilon$ does not change the leading asymptotic behaviour $\sim \frac{1}{l^{3}}$ of the series expansion of the redefinition (5). This implies that the specification of an asymptotic behaviour for the coefficients $a_{l}$ is not sufficient to specify a proper functional class beyond the proposal of [6]. Some further condition on the redefinitions must be introduced in order recover a unique admissible choice for $\alpha_{s}$ when enlarging the functional space beyond the proposal of [5, 6].

Notice that the above analysis of the coefficients has a simple interpretation. Given a certain field redefinition with coefficients $a_{l}$ bound to have a certain asymptotic behaviour, the corresponding improvement can be obtained by a simple action of the covariant adjoint derivative whose effect is to produce some other pseudo-local tail with coefficients $\tilde{a}_{l}$ which can be expressed linearly in terms of the coefficients $a_{l}, a_{l-1}$ and $a_{l-2}$ [6]:

$$
\tilde{a}_{l}=A_{l}^{(s)} a_{l}+B_{l-1}^{(s)} a_{l-1}+C_{l-2}^{(s)} a_{l-2} .
$$

This means that $\tilde{a}_{l} \prec a_{l}$. On the other hand it is impossible to obtain coefficients $\tilde{a}_{l}$ growing for $l \rightarrow \infty$ more slowly than the original set of coefficients $a_{l}$. The only possibility is to have some finetuning so that the coefficients $\tilde{a}_{l}$ go much faster to zero than the original coefficients. This implies that in order to remove by a field redefinition a backreaction with a given asymptotic behaviour for its coefficients the best one can do is to have a redefinition with the same asymptotic behaviour $\tilde{a}_{l} \sim a_{l}$. This simple argument indirectly implies that the redefinition proposed in [11] should also be compatible with the asymptotic behaviour presented in this note. In more detail, a simple test of the functional class proposal of $[11,12]$ would be to check if the redefinitions (57) for different values of $\alpha_{s}$ than (6) are indeed not admissible.

- It might be of some interest also to consider a different perspective on the same problem. It is indeed possible to avoid to talk about the subtle issue of field redefinitions and study the limit of the finite derivative truncations of a given back-reaction:

$$
J=\sum_{l=0}^{\infty} \frac{a_{l}}{l ! l !} \square^{l}(\nabla \ldots \nabla \phi \nabla \ldots \nabla \phi) \equiv \lim _{k \rightarrow \infty} \underbrace{\sum_{l=0}^{k} \frac{a_{l}}{l ! l !} \square^{l}(\nabla \ldots \nabla \phi \nabla \ldots \nabla \phi)}_{J_{k}} .
$$

In the above procedure each finite-derivative truncation is well-defined and one can extract the canonical-current piece of each truncation unambiguously. Analogously, one can compute for each truncation the corresponding Witten diagram using standard techniques from local field theories and take the limit only afterwards $[5,6]$. 
We declare that the limit exists when the limit is finite (no infinite coefficient is generated) and is independent of local redefinitions $f_{k}$ or $g_{k}$ performed on each given truncation under the assumption that they converge to admissible redefinitions $f_{\infty}$ and $g_{\infty}$ according to ${ }^{5}$ [6]. Using a diagrammatic language, the existence of the limit can be summarised by the following commutative diagram:

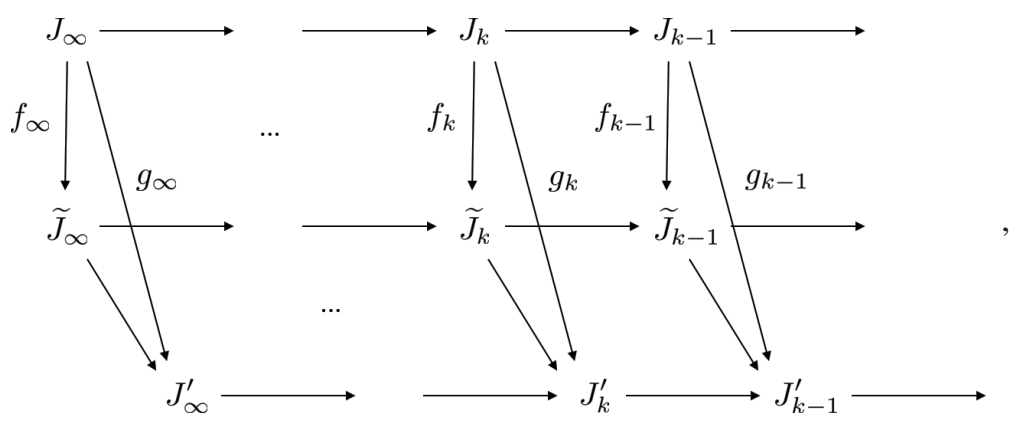

where $f_{\infty}$ and $g_{\infty}$ belong to the functional class defined in [6] while $\widetilde{J}_{k}$ and $J_{k}^{\prime}$ are different local forms of the truncation which differ by a local field redefinition.

If this limit exists we can resum the higher-derivative tail and extract the coefficient of the canonical Metsaev-like coupling. If the limit does not exist, as observed in [5] for the backreaction (1), it might still be possible to define the sum via some analytic continuation. This is a standard situation where one can define the sum of infinite series formally introducing a cut off procedure. An example of this procedure is:

$$
\sum_{l=0}^{\infty} 1
$$

which can be regularised introducing a cut-off as:

$$
\sum_{l=0}^{\infty} e^{-\epsilon(l+\Lambda)}=\frac{e^{\epsilon-\Lambda \epsilon}}{e^{\epsilon}-1} \sim \frac{1}{\epsilon}+\left(\frac{1}{2}-\Lambda\right)+O(\epsilon) .
$$

As expected, the finite part of the result is cut-off dependent and hence ambiguous. For a backreaction (8) with a divergent sum one similarly ends up with expressions which can be defined formally by analytic continuation. For each given spin there exist a choice of cut-off which reproduces the result expected by the holographic reconstruction (6). The question then becomes the same which is usually asked about a renormalisable theory. Namely, whether the choice of regularisation which gives results compatible with holographic reconstruction (6) is spin-dependent. If the choice of cut-off is spin independent, the choice for spin 2 will fix at the same time the whole backreaction unambiguously. However, if the proper choice of cut-off compatible with the holographic reconstruction is spin-dependent the corresponding analytic continuation is not predictive.

\footnotetext{
${ }^{5}$ We briefly recall that in order to check whether a redefinition $f_{\infty}$ belongs to the functional class of [6] one first needs to compute the associated improvement $J^{(f)}$ generated by the field redefinition at this order. The corresponding redefinition is then considered admissible iff the limit of the projections of each local truncation of $J^{(f)}$ on the local canonical coupling is vanishing:
}

$$
\lim _{k \rightarrow \infty} J_{k}^{(f)}=\left(\lim _{k \rightarrow \infty} a_{k}\right) \mathbf{J}+\lim _{k \rightarrow \infty} \Delta_{k}, \quad \quad \lim _{k \rightarrow \infty} a_{k}=0 .
$$

Here $\mathbf{J}$ is a fixed, but otherwise arbitrary, (local) representative for the non-trivial canonical coupling. 
In the following we give the regularised results for the backreaction for $s=2,4,6$ using the results of $[5,6]$ :

$$
\begin{aligned}
\alpha_{2}(\Lambda) & =\frac{1}{36}\left(1-6 \Lambda^{2}\right) \\
\alpha_{4}(\Lambda) & =\frac{-2100 \Lambda^{6}+14280 \Lambda^{5}-31290 \Lambda^{4}+26600 \Lambda^{3}-1680 \Lambda^{2}+10080 \Lambda+34567}{1058400}, \\
\alpha_{6}(\Lambda) & =\frac{1}{92207808000}\left(-291060 \Lambda^{10}+6338640 \Lambda^{9}-57387330 \Lambda^{8}+280637280 \Lambda^{7}\right. \\
& -802849740 \Lambda^{6}+1351860048 \Lambda^{5}-1257850440 \Lambda^{4}+525866880 \Lambda^{3} \\
& \left.-3991680 \Lambda^{2}+79833600 \Lambda+415046341\right) .
\end{aligned}
$$

It is now easy to verify that the choice of the cut-off which matches the holographically reconstructed result (6), in the appropriate normalisation, for $\alpha_{2}$ is not consistent with $\alpha_{4}$ or $\alpha_{6}$ with the same normalisation. Therefore, the cut-off $\Lambda$ must be spin-dependent, to compensate the highly spin-dependent form of the above regularised expressions. This makes the corresponding analytic continuations unpredictive.

This result is not in contradiction with the analysis of field redefinitions presented in this note. The above feature may be a different reincarnation of the fact that all redefinitions removing the higherderivative tail, regardless the value of $\alpha_{s}$, have the same asymptotic behaviour at $l \rightarrow \infty$. Similarly, this does not allow to single out a unique value of $\alpha_{s}$.

- It might be interesting to compare the complicated redefinition (5) which matches the holographically reconstructed result starting from (1) with other redefinitions which would generate the required coupling constant but from a free theory. One may indeed start with free Fronsdal equations and find the non-local redefinition which would generate the appropriate cubic couplings. This redefinition should not be admissible but, remarkably, it has a faster asymptotic behaviour for $l \rightarrow \infty$ than (5):

$$
a_{l-1}=\alpha_{s} s !(s-1) !(2 l+s+2)\left(\frac{l !}{(l+s+1) !}\right)^{2} .
$$

This expression is simpler than (5), and falls off faster as $l \rightarrow \infty: a_{l} \sim \frac{1}{l^{2 s+1}}$. Notice that the above redefinition (16) allows to generate the holographic backreaction from the free theory with the choice (6). The above redefinition however should not be considered admissible as it does not leave the cubic Witten diagrams invariant.

- In the parity violating case, the backreaction (1) is multiplied by a factor proportional to the parity violating phase $\theta$. Surprisingly (see $[3,18]$ ) this factor is given by $\cos (2 \theta)$ :

$$
J_{s}(\theta)=\sum_{l=0}^{\infty} \frac{j_{l} \cos (2 \theta)}{l ! l !} \square^{l}(\nabla \ldots \nabla \phi \nabla \ldots \nabla \phi)_{s},
$$

It was then observed in [3] that term by term each element of the pseudo-local series in (1) vanishes identically for $\theta=\frac{\pi}{4}$. The interpretation of this $\theta$-dependence is at the moment unclear as it seems to be in contraddiction with the holographic expectations [19]. In [11] it was proposed that up to an admissible field redefinition one is left with a canonical current and a $\theta$-independent coefficient. Let us assume that the field redefinition reproducing the holographically expected coupling constants is admissible. This means that the difference between the above non-local current (17) and a local 
canonical current with a fixed non-vanishing coefficient ${ }^{6} \alpha_{s}$ is an admissible improvement:

$$
J_{s}(\theta)-\alpha_{s} j_{s}^{\text {can. }}=\Delta_{s}^{(J)}(\theta) .
$$

Since the above must be true for any value of $\theta$, we can now set $\theta=\frac{\pi}{4}$ and use that $J_{s}\left(\frac{\pi}{4}\right)=0$. This however implies that the canonical current itself is an admissible improvement:

$$
\alpha_{s} j_{s}^{\text {can. }}=\Delta_{s}^{(J)}\left(\frac{\pi}{4}\right),
$$

This further implies that $\Delta J_{s}(\theta)+x \Delta_{s}^{(J)}\left(\frac{\pi}{4}\right)$ is an admissible improvement whose associated admissible field redefinition puts any coefficient (parameterised by $x$ ) in front of the canonical current. This contraddicts our assumption on the admissibility of the above field-redefinition. Furthermore, the above shows that in the $\theta=\frac{\pi}{4}$ case the initial redefinition itself must generate the canonical backreaction from the free Fronsdal equations, and therefore has to match (16). Let us also stress that the only assumptions we used is the existence of a field redefinition which is both allowed and capable of changing the $\theta$ dependence of the initial pseudo-local current (17). The contraddiction we find seems to imply that no such admissible redefinition may exist.

This note is organised as follows. After a brief review of the main formalism in Section 2, we move to the analysis of the redefinitions and to the study of their structure in Section 3. We end with a short summary and some outlook in Section 5.

\section{Pseudo-local currents}

In $[2,3,5,6,10]$ a convenient generating function formalism was developed, first in $3 \mathrm{~d}$ and then in $4 \mathrm{~d}$, to manipulate quasi-local current interactions and corresponding field redefinitions. In this section we recall the basic ingredients of the formalism, and refer to $[2,5]$ for further details. The main object is the zero-form $C(y, \bar{y} \mid x)$, which is a formal expansion in the spinorial oscillators $y_{\alpha}$ and $\bar{y}_{\dot{\alpha}}$ satisfying the linearised unfolded equations

$$
\widetilde{D} C(y, \bar{y} \mid x)=0 .
$$

Here $D$ and $\widetilde{D}$ are the adjoint and twisted-adjoint covariant derivatives expressed in terms of spinorial oscillators as: ${ }^{7}$

$$
\begin{aligned}
& D=\nabla-h^{\alpha \dot{\alpha}}\left(y_{\alpha} \bar{\partial}_{\dot{\alpha}}-\bar{y}_{\dot{\alpha}} \partial_{\alpha}\right), \\
& \widetilde{D}=\nabla+i h^{\alpha \dot{\alpha}}\left(y_{\alpha} \bar{y}_{\dot{\alpha}}-\partial_{\alpha} \bar{\partial}_{\dot{\alpha}}\right) .
\end{aligned}
$$

In the unfolded language, the zero-form is the main ingredient upon which one constructs ordinary current interactions. Furthermore, upon solving the twisted adjoint covariant constancy condition one recovers the relations between components of the zero form and derivatives of the linearised Weyl tensors associated to the HS fields:

$$
C_{\alpha(k+2 s), \dot{\alpha}(k)}(x) \sim \nabla^{k} C_{\alpha(2 s)}(x), \quad \quad C_{\alpha(k), \dot{\alpha}(k+2 s)}(x) \sim \nabla^{k} C_{\dot{\alpha}(2 s)}(x),
$$

Above, $C_{\alpha(2 s)}(x)$ and $C_{\dot{\alpha}(2 s)}(x)$ are the self-dual and anti-self dual part of the linearised HS Weyl tensor for $s>0$ and $\Phi(x) \equiv C(0,0 \mid x)$ is the actual scalar field.

\footnotetext{
${ }^{6}$ Whether or not $\alpha_{s}$ depends on $\theta$ is not important for the following argument. The only assumption is that $\alpha_{s}$ does not vanish for any value of $\theta$.

${ }^{7} h^{\alpha \dot{\alpha}}$ is the vierbein, $\nabla=d-\omega^{\alpha \alpha} y_{\alpha} \partial_{\alpha}-\varpi^{\dot{\alpha} \dot{\alpha}} \bar{y}_{\dot{\alpha}} \bar{\partial}_{\dot{\alpha}}$ is the $\operatorname{AdS}_{4}$ covariant derivative, while $\varpi^{\alpha \alpha}$ and $\varpi^{\dot{\alpha} \dot{\alpha}}$ are the (anti-)selfdual components of the spin-connection of $\mathrm{AdS}_{4}$.
} 
A current which sources the HS Fronsdal operator is a bilinear functional $J_{\alpha(s) \dot{\alpha}(s)}(C, C)$ of the zero-form $C$, which is conserved on the equations of motion (20):

$$
\nabla^{\beta \dot{\beta}} J_{\beta \alpha(s-1) \dot{\beta} \dot{\alpha}(s)} \approx 0 .
$$

For practical purposes, it is convenient to specify the most general form for a current in terms of a generating function $\operatorname{Kernel} J(Y, \xi, \eta)$ :

$$
\mathbf{J}(C, C)=\int d^{4} \xi d^{4} \eta J(Y, \xi, \eta) C(\xi \mid x) C(\eta \mid x) .
$$

Above we have introduced the Fourier transform of the 0-form with respect to the spinorial variables $y$ and $\bar{y}$,

$$
C(y, \bar{y} \mid x)=\int d^{2} \xi d^{2} \bar{\xi} e^{i y^{\alpha} \xi_{\alpha}+i \bar{y} \bar{\xi}^{\dot{\alpha}} \bar{\xi}_{\dot{\alpha}}} C(\xi, \bar{\xi} \mid x),
$$

where for ease of notation we denote both Fourier transforms and original 0-forms by the same letter. In this way, contractions of indices are encoded as simple monomials:

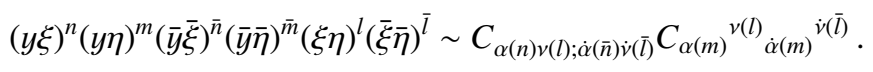

In addition to the above way of representing a generic current interaction it is also convenient to introduce generating functions of coefficients via contour integrals, as originally proposed in [10]. Restricting the attention to the canonical current sector, which in four-dimensions is uniquely specified by the absence of trace components, one can then write the most general current kernel as

$$
J^{\text {can. }}(Y, \xi, \eta)=\oint_{\tau_{i}, s, r} g\left(\alpha_{1}, \alpha_{2}, \beta, \gamma\right) e^{i\left(s y \zeta^{-}+\tau_{1} \xi \eta+r \bar{y} \bar{\zeta}^{+}+\tau_{2} \bar{\xi} \bar{\eta}\right)}
$$

in terms of a function of 4 complex variables:

$$
\begin{aligned}
& \alpha_{1}=\tau_{1}^{-1}, \\
& \alpha_{2}=\tau_{2}^{-1}, \\
& \beta=s^{-1}, \\
& \gamma=r^{-1}, \\
& \zeta^{ \pm}=\xi \pm \eta, \\
& \bar{\zeta}^{ \pm}=\bar{\xi} \pm \bar{\eta} .
\end{aligned}
$$

These parametrise the four contractions of indices relevant to the canonical current sector in $4 \mathrm{~d}$. One can easily translate between the contour integral form and the generating function form via:

$$
\alpha_{2}^{m+1} \alpha_{1}^{n+1} \beta^{s_{1}+1} \gamma^{s_{2}+1} \rightarrow \frac{(i \bar{\xi} \bar{\eta})^{m}(i \xi \eta)^{n}\left(i \bar{y} \bar{\zeta}^{+}\right)^{s_{2}}\left(i y \zeta^{-}\right)^{s_{1}}}{m ! n ! s_{1} ! s_{2} !} .
$$

Notice that in this generating function calculus the function $g\left(\alpha_{1}, \alpha_{2}, \beta, \gamma\right)$ is defined as a formal series, and this should be thought at this level as a regularity condition. This regularity condition will be assumed here since it is equivalent to pseudo-locality of the back-reaction. Notice also that a constant term or terms proportional to $1 / \alpha_{1}$ drop out of the contour integration. In particular, two generating functions will give the same pseudo-local current if they differ by terms of this type. For the details we refer to $[2,10]$ and use the symbol $\sim$ to indicate equality modulo the above equivalence relation.

To conclude this section we present the corresponding expressions for the explicit Fronsdal currents of the type $s-s_{1}-s_{2}$ :

$$
\frac{1}{4(s-1)} \underbrace{\left[\square+2\left(s^{2}-2 s-2\right)+\ldots\right] \phi}_{\mathcal{F}}=J^{\mathrm{Fr} .},
$$


as extracted from Vasiliev's equations ${ }^{8}$ in [3]:

$$
J^{\mathrm{Fr} .}=\frac{i}{2} e^{2 i \theta}(\beta \gamma)^{s+1} \sum_{n, m} c_{n, m}^{(s)}\left(\frac{p_{1}(n)-m p_{2}(n)}{(n+s-1)^{2}(n+s)^{2}(n+s+1)}\right) \alpha_{1}^{n} \alpha_{2}^{m}+\text { h.c. },
$$

with

$$
\begin{aligned}
p_{1}^{(s)}(n) & =\left[[2(s-1)] n^{3}+[2+s(6 s-5)] n^{2}+\left[s\left(1-4 s+6 s^{2}\right)\right] n\right. \\
& +[s(1+s)(2+s(2 s-3))]], \\
p_{2}^{(s)}(n) & =\left[[2(s-1)] n^{2}+[2+s(4 s-3)] n+s(1+s(2 s-1))\right],
\end{aligned}
$$

and

$$
c_{n, m}^{(s)}=\frac{1}{4(s-1)} \frac{(-1)^{m+s}+(-1)^{n}}{2} .
$$

Notice that powers of $\alpha_{1}$ and $\alpha_{2}$ translate into contractions among the 0 -form, hence powers of $\alpha_{1} \alpha_{2}$ parameterise powers of $\square$ in the metric-like language. We can then define $\tau=\alpha_{1} \alpha_{2}$ parametrising the pseudo-local tail of the given interaction term. In general, when restricting attention to sources to the Fronsdal equation in the spin-s sector, one fixes the dependence on $\beta$ and $\gamma$ as in (33) while working with a generating function of the type:

$$
J_{s}=i \cos (2 \theta)(\beta \gamma)^{s+1} \alpha_{1}^{n} g(z), \quad z=\alpha_{1} \alpha_{2} .
$$

The function $g(z)$ parameterises the infinite non-local tail while the dependence on $\beta, \gamma$ and a single $\alpha_{1}$ fixes the canonical current tensor structure. In the $s-0-0$ case the dictionary can be given quite explicitly as:

$$
\begin{aligned}
J_{s} & \rightarrow \cos (2 \theta) \sum_{l, k} a_{l, k} \nabla_{\mu(s-k) v(l)} \Phi \nabla_{\mu(k)}{ }^{v(l)} \Phi, \\
\nabla_{\mu(s-k) v(l)} \Phi \nabla_{\mu(k)}{ }^{v(l)} \Phi & \equiv h_{\mu}^{\alpha \dot{\alpha}} \ldots h_{\mu}^{\alpha \dot{\alpha}} C_{\alpha(s-k) v(l), \dot{\alpha}(s-k) \dot{v}(l)} C_{\alpha(k)}{ }_{, \dot{\alpha}(s-k)}{ }^{\nu(l)},
\end{aligned}
$$

with

$$
a_{l, k}=\frac{(-1)^{k+l} s ! s !}{l ! l ! k ! k !(s-k) !(s-k) !} g_{l}, \quad g(z)=\sum_{l=0}^{\infty} g_{l} z^{l+1}
$$

The above dictionary holds also for redefinitions of the Fronsdal field and allows to easily translate from the generating function language to the standard tensorial language. Notice that the notation $\nabla_{\mu(s-k) v(l)} \Phi \nabla_{\mu(k)}{ }^{v(l)} \Phi$ is defined by eq. (39) and includes symmetrisation and traceless projection. In particular $\nabla_{\mu(s)} \neq \nabla_{\mu} \ldots \nabla_{\mu}$. We give further details in Appendix A.

In the following we shall restrict our attention to the function $g(z)$ while assuming the above dictionary to go back to standard tensorial notation. It is important to stress that the $\frac{1}{(l !)^{2}}$ factor in (40) arises via the above contour integrations and does not appear in the function $g(z)$.

\footnotetext{
${ }^{8}$ The normalisation for the Fronsdal tensor comes from the solution to torsion as described in [3].
} 


\section{Pseudo-Local Field Redefinitions}

In this section we employ the generating function formalism to study the non-local field redefinitions which relate the back-reaction extracted from Vasiliev's equation in [3] to canonical currents. For simplicity we work in the A-type model, setting $\theta=0$. The discussion generalises straightforwardly to any choice of $\theta$ as this only appears as an overall factor. The effect of a field redefinition of the spin- $s$ field quadratic in the 0 -form can be encoded in an arbitrary function $k(z)$ (analogous to $g(z)$ above) via the differential operator ${ }^{9}$

$$
\begin{aligned}
\delta J_{s}=\frac{(\beta \gamma)^{s+1}}{4(s-1)}\left[z^{2}(z+1)^{2} k_{s}^{\prime \prime}(z)+\right. & z(z+1)(2 s+(3+\bar{n}) z+3+\bar{n}) k_{s}^{\prime}(z) \\
& \left.+\left(\bar{n}(z+1)(z+s+1)+s^{2}(z+1)+2 s+(z+1)^{2}\right) k_{s}(z)\right],
\end{aligned}
$$

where $\bar{n}=n-m$ with $n$ and $m$ giving the power of $\alpha_{1}$ and $\alpha_{2}$ respectively as in (33). In this way, the problem of finding redefinitions which remove the pseudo-local tail is thus mapped into a ordinary differential equation.

In the following we apply this formalism to the current (33). We first give the explicit field redefinition which maps the pseudo-local current (33) to a canonical current with an arbitrary overall coefficient:

$$
\underbrace{\square \phi+\ldots}_{\mathcal{F}}=\alpha_{s} J^{\mathrm{can}} .
$$

We then compare redefinitions which give different overall coefficients $\alpha_{s}$ for the canonical current. For ease of notation and without loss of generality we restrict the attention to the currents with $\bar{n}=0$.

\section{Spin-2:}

Using the generating function formalism reviewed above, the back-reaction (33) for $s=2$ and $\bar{n}=0$ can be encoded by (see eq. (37)):

$$
g_{2}(z)=\sum_{l=0}^{\infty} \frac{(-1)^{l}}{2(l+1)^{2}(l+2)} z^{l}=\frac{z-z \operatorname{Li}_{2}(-z)-(z+1) \log (z+1)}{2 z^{2}}
$$

The field redefinition that reduces the above pseudo-local current to its canonical form can be obtained solving the differential equation:

$$
z(z+1)\left(z(z+1) k_{2}^{\prime \prime}(z)+(3 z+7) k_{2}^{\prime}(z)\right)+(z+3)^{2} k_{2}(z)=-4 g_{2}(z)+\alpha_{2} z+\beta_{2} .
$$

The constant $\beta_{2}$ parameterises non-trivial pseudo-local redefinitions which result in a vanishing ${ }^{10}$ contribution to the backreaction upon performing the contour integration. On the other hand, $\alpha_{2}$ is an arbitrary constant in front of the canonical current (which in this case is the stress tensor (43)). In the following we fix the constant $\beta_{2}=2 \alpha_{2}+2$ in such a way that the slowest contribution to the coefficients in the $l \rightarrow \infty$ limit is set to zero. Furthermore, one should carefully pick the unique

${ }^{9}$ The corresponding redefinition at the level of unfolded equations reads:

$$
\delta \omega_{s}(y, \bar{y})=h^{\alpha \dot{\alpha}} \partial_{\alpha} \bar{\partial}_{\dot{\alpha}} k_{s}(y, \bar{y}) .
$$

\footnotetext{
${ }^{10} \mathrm{We}$ have not considered terms on the right-hand side of the type $z^{-m}$, since the corresponding redefinitions vanish upon performing the contour integration. This can be checked by solving the differential equation (see [2]).
} 
solution to the above differential equation which is analytic in $z$, as this corresponds to the standard regularity condition on pseudo-local functionals $[5,10]$. The final result has the following series expansion around $z=0$ :

$$
k_{2}(z)=\sum_{l=0}^{\infty} \frac{\left(4 \alpha_{2}+l(l+3)+2\right)}{(l+1)^{2}(l+2)(l+3)^{2}}(-z)^{l},
$$

where we have left $\alpha_{2}$ arbitrary.

Spin-4:

The source to the spin-4 Fronsdal operator extracted from (33) for $\bar{n}=0$ can be encoded in the following generating-function:

$$
g_{4}(z)=\sum_{n=0}^{\infty} \frac{(3 l+11)}{3(l+3)^{2}(l+4)^{2}}(-z)^{l} \sim-\frac{1}{3 z^{4}}\left[(2 z+1) \operatorname{Li}_{2}(-z)+(z+1) \log (z+1)\right] .
$$

The redefinition bringing the above back-reaction to its canonical form (43) with an arbitrary coefficient $\alpha_{4}$, can be obtained solving the differential equation:

$$
\begin{aligned}
z(z+1)\left(z(z+1) k_{4}^{\prime \prime}(z)+(3 z+11) k_{4}^{\prime}(z)\right)+\left(z^{2}+18 z+25\right) k_{4}(z) & =-12 g_{4}(z)+\alpha_{4} z+\beta_{4} .
\end{aligned}
$$

Again, $\beta_{4}$ parameterises pseudo-local redefinitions which do not change the back-reaction, and can be chosen to improve the asymptotic behaviour. In this case we choose $\beta_{4}=\frac{1}{2}\left(3 \alpha_{4}+4\right)$. The solution to the above differential equation is then:

$$
k_{4}(z)=\sum_{l=0}^{\infty} \frac{2}{5} \frac{p_{4}(l)+120\left(6 \alpha_{4}+5\right)}{(l+1)^{2}(l+2)^{2}(l+3)(l+4)^{2}(l+5)^{2}}(-z)^{l},
$$

with $p_{4}(l)$ a polynomial of order 5 in the variable $l$ :

$$
p_{4}(l)=l\left(5 l^{5}+77 l^{4}+470 l^{3}+1445 l^{2}+2345 l+1898\right) .
$$

Spin-6:

The source to the spin-6 Fronsdal operator extracted from (33) for $\bar{n}=0$ can be encoded in the generating-function

$$
\begin{aligned}
g_{6}(z)=\sum_{l=0}^{\infty} \frac{3(5 l+28)}{10(l+5)^{2}(l+6)^{2}}(-z)^{l} & \\
& \sim-\frac{3}{10 z^{6}}\left[(z+1) \log (z+1)+(3 z+2) \operatorname{Li}_{2}(-z)\right] .
\end{aligned}
$$

The redefinition bringing the above back-reaction to its canonical form (43) with an arbitrary coefficient $\alpha_{6}$ can be obtained solving the differential equation:

$$
\begin{aligned}
z(z+1)\left(z(z+1) k_{6}^{\prime \prime}(z)+3(z+5) k_{6}^{\prime}(z)\right)+z(z+38)+49 k_{6}(z) \quad=-20 g_{6}(z)+\alpha_{6} z+\beta_{6} .
\end{aligned}
$$


Again, $\beta_{6}$ parameterises pseudo-local redefinitions which do not change the back-reaction. In this case we choose $\beta_{6}=\frac{1}{3}\left(4 \alpha_{6}+6\right)$. The solution to the above differential equation is then given by:

$$
k_{6}(z)=\sum_{l=0}^{\infty} \frac{p_{6}(l)+2 \cdot 6 ! \cdot 5 !\left(\alpha_{6}+1\right)}{(l+1)^{2}(l+2)^{2}(l+3)^{2}(l+4)(l+5)^{2}(l+6)^{2}(l+7)^{2}}(-z)^{l},
$$

with $p_{6}(l)$ a polynomial of order 10 in the variable $l$ :

$$
\begin{aligned}
p_{6}(l)=l\left(3 l^{9}+\frac{323 l^{8}}{3}+1686 l^{7}+\frac{105862 l^{6}}{7}\right. & +85755 l^{5}+320009 l^{4} \\
& \left.+792684 l^{3}+\frac{3844612 l^{2}}{3}+1288512 l+\frac{5072640}{7}\right) .
\end{aligned}
$$

Generic even spin:

In the generic spin- $s$ case, the source quadratic in the 0-form to the Fronsdal operator extracted in [3] can be encoded by the generating-function

$$
\begin{aligned}
g_{s}(z)=\sum_{l=0}^{\infty} \frac{s(2 l(s-1)+s(2 s-3)+2)}{8(s-1)(l+s-1)^{2}(l+s)^{2}} & (-z)^{n} \\
& \sim-\frac{c_{s}}{z^{s}}\left[(z+1) \log (z+1)+\frac{1}{2}(s(z+1)-2) \operatorname{Li}_{2}(-z)\right],
\end{aligned}
$$

with

$$
c_{s}=\frac{1}{4} \frac{s}{s-1} .
$$

The corresponding redefinition mapping the above pseudo-local back-reaction to a canonical current (43) with overall coefficient $\alpha_{s}$ (43) is:

$$
k_{s}(z)=\sum_{l=0}^{\infty} \frac{2 l+s+2}{2}\left(\frac{l !}{(l+s+1) !}\right)^{2}\left[p_{s}(l)+a_{s} \alpha_{s}\right](-z)^{l},
$$

with $p_{s}(l)$ a polynomial of degree $2(s-1)$ in the variable $l$ and $a_{s}$ a spin-dependent constant. The general form of the polynomial for arbitrary spin is cumbersome, and we do not present it explicitly.

\section{Analytic continuation}

In this section we study the analytic continuation of the formal series obtained by considering the limit of the lower derivative truncations of the backreaction (1).

The main observation of $[5,6]$ is that for any truncation of the pseudo-local interaction term (33) it is possible to compute the corresponding projection on the canonical current piece which is parametrised by finitely many structures in correspondence with Metsaev's cubic couplings. Each higher-derivative term gives a contribution to the canonical coupling weighted by some proportionality factor $C_{l}^{(s)}$ which measures the overlap of the higher-derivative term on the canonical structure. Schematically the projection reads:

$$
\mathcal{P}\left(\square^{l}(\nabla \ldots \nabla \phi \nabla \ldots \nabla \phi)_{\mu(s)}\right)=C_{l}^{(s)}(\nabla \ldots \nabla \phi \nabla \ldots \nabla \phi)_{\mu(s)},
$$


where indeed the right-hand side is proportional to the canonical structure times a certain overall coefficient which was computed in $[5,6]$.

After the projection the canonical structure factorises and one is left with an overall coefficient which combines together all contributions from each higher-derivative term. Below we give the corresponding coefficient for some low spin examples:

$$
\begin{array}{ll}
s=2, & -\frac{1}{12} \sum_{l=1}^{\infty} l, \\
s=4, & -\frac{1}{3 \cdot 7 !} \sum_{l=1}^{\infty} \frac{l(l+1)(l+2)^{2}(3 l+11)(5 l(l+4)+3)}{(l+3)(l+4)} \\
s=6, & -\frac{3}{5 \cdot 11 !} \sum_{l=1}^{\infty} \frac{(l+4) !}{(l-1) !} \frac{(l+3)(5 l+28)(7 l(l+6)(3 l(l+6)+19)+20)}{(l+5)(l+6)}, \\
s=8, & -\frac{7}{6 \cdot 15 !} \sum_{l=1}^{\infty} \frac{(l+6) !}{(l-1) !} \frac{(l+4)(7 l+53)(l(l+8)(11 l(l+8)(13 l(l+8)+274)+14631)+420)}{(l+7)(l+8)} .
\end{array}
$$

These series are divergent and can be regularised by a standard $\zeta$-function regularisation introducing a regulator of the type $e^{-\epsilon(l+\Lambda)}$ which allows to resum them. Dropping the divergent parts and setting $\epsilon$ to zero one then obtains the following regularised expressions for the corresponding overall coefficients of the canonical current: ${ }^{11}$

$$
\begin{aligned}
\alpha_{2}(\Lambda) & =\frac{1}{36}\left(1-6 \Lambda^{2}\right) \\
\alpha_{4}(\Lambda) & =\frac{-2100 \Lambda^{6}+14280 \Lambda^{5}-31290 \Lambda^{4}+26600 \Lambda^{3}-1680 \Lambda^{2}+10080 \Lambda+34567}{1058400} \\
\alpha_{6}(\Lambda) & =\frac{1}{92207808000}\left(-291060 \Lambda^{10}+6338640 \Lambda^{9}-57387330 \Lambda^{8}+280637280 \Lambda^{7}\right. \\
& -802849740 \Lambda^{6}+1351860048 \Lambda^{5}-1257850440 \Lambda^{4}+525866880 \Lambda^{3} \\
& \left.-3991680 \Lambda^{2}+79833600 \Lambda+415046341\right) \\
\alpha_{8}(\Lambda) & =\frac{1}{39269461271040000}\left(-51531480 \Lambda^{14}+2307024720 \Lambda^{13}-45901996140 \Lambda^{12}\right. \\
& +535422167280 \Lambda^{11}-4065466421016 \Lambda^{10}+21086692426800 \Lambda^{9} \\
& -76212086580630 \Lambda^{8}+191985197049360 \Lambda^{7}-330826659683520 \Lambda^{6} \\
& +372883114251648 \Lambda^{5}-249836835568320 \Lambda^{4}+77398236115200 \Lambda^{3} \\
& \left.-62270208000 \Lambda^{2}+2615348736000 \Lambda+16930453296697\right) .
\end{aligned}
$$

These results allow in principle to fix the cutoff in order to recover the holographically reconstructed result of $[7,13]$. The main observation is however that the associated choice for the cutoff is spindependent. Furthermore the regularised coupling constant is given by complicated polynomials of order $\Lambda^{2(s-1)}$. The lack of a spin-independent regularisation makes the corresponding analytic continuations unpredictive as a consequence of the strongly coupled nature of the higher-derivative tails. It is therefore not clear how to identify a regularisation which preserves all HS symmetries without

\footnotetext{
${ }^{11}$ In the formulas for $\alpha_{s}(\Lambda)$ we take into account the normalisation $\frac{1}{4(s-1)}$ in (32) so to arrive to a source of the type $\mathcal{F}_{\mu(s)}=$ $\alpha_{s}(\Lambda) J_{\mu(s)}^{\text {can. }}$.
} 
solving the Noether procedure up to the quartic order. On the other hand, the simple form of the local holographically reconstructed couplings [7] predicts a very simple structure for the corresponding local interactions.

\section{Conclusions}

In this note we have determined explicitly the general form of the redefinition which reduces the backreaction extracted in [3] from Vasiliev's equations to a canonical current with an arbitrary overall coefficient (43). We have also given some details about the analytic continuation of the formally divergent sums. Our analysis provides a convenient test ground to probe functional class proposals for admissible non-local interactions.

The main conclusion of this note is that the dictionary between Vasiliev's equations and standard HS equations like Fronsdal's equations will not be complete unless a full non-perturbative functional class able to fix the overall coefficient in front of the canonical interaction terms will be provided. Furthermore, it is worth stressing that in this note we have restricted the attention to the cubic order which admits a local completion at least for fixed spins. At the quartic and higher orders we expect the situation to be even more subtle, since non-localities may not be anymore removed by redefinitions, and very restrictive consistency requirements will be put in place by consistency $[6,20]$.

We conclude this note with a short list of observations and comments:

- The main difficulty of HS theories is the absence of a scale beyond the AdS-radious. This implies that the behaviour of a pseudo-local tail can only be controlled by its coefficients owing to $\Lambda[\nabla, \nabla] \sim$ 1. A functional class proposal would then prescribe a given asymptotic behaviour for the coefficients of higher and higher order terms as in the proposal of [6].

- It would be nice to study the limit $\alpha^{\prime} \rightarrow \infty$ at the level of effective cubic string field theory couplings in AdS. Some issues about commutativity of limits may arise in this context when taking the limit $\alpha^{\prime} \rightarrow \infty$ before or after removing the higher-derivative tails. It might well be that if one considers the naive $\alpha^{\prime} \rightarrow \infty$ limit of a non-local string coupling this would indeed be strongly coupled. This would mean that the tensionless limit should be well-defined only in a particular field frame, while singular in others. This kind of situation suggests that allowed redefinitions for any finite value of $\alpha^{\prime}$ could become not allowed after the limit. If so the infinities observed in this note could be resolved upon taking the limit from string theory using $\alpha^{\prime}$ as regulator for the strongly coupled series.

- Beyond the particular problem of mapping the tail in (1) to a canonical form, the simplicity of the $4 \mathrm{~d}$ theory in the spinorial language is expected to manifest in a simple form of all quadratic sources when rewritten in the unfolded form. Such rewriting, and the structures involved beyond the $\star-$ product, is at the moment unknown (see however [21, 22]). For this reason an interesting problem would be to unfold the non-linear Fronsdal equations in 4d coming from the cubic couplings extracted holographically in [7]. This result would provide us with the complete list of cubic vertices in the unfolded equations possibly giving us a hint of higher-order completions.

\section{Acknowledgments}

I am grateful to N. Boulanger, S. Didenko, D. Francia, A. Sagnotti, E. Sezgin, C. Sleight, Z. Skvortsov, D. Sorokin, P. Sundell and M. Vasiliev for useful discussions. The research of M. Taronna is partially supported by the Fund for Scientific Research-FNRS Belgium, grant FC 6369 and by the Russian Science Foundation grant 14-42-00047 in association with Lebedev Physical Institute. This research was also supported by the Munich Institute for Astro- and Particle Physics (MIAPP) of the DFG cluster of excellence "Origin and Structure of the Universe". 


\section{A Canonical Currents Quadratic in the Curvatures}

In this appendix we would like to give a few more details on the explicit tensorial form of the currents discussed in this note. Canonical currents are encoded as generating functions by $(\beta \gamma)^{s+1} \alpha_{1}^{n} \tau$, since any dependence on $\alpha_{1} \alpha_{2}$ can be removed by a local field redefinition. Such monomial corresponds to the following generating function kernels:

$$
\begin{aligned}
(\beta \gamma)^{s+1} \alpha_{1}^{n} \tau \rightarrow \frac{1}{s !^{2} n !}\left(-i \partial_{\xi} \cdot \partial_{\eta}\right)^{n}\left(-i y\left(\partial_{\xi}-\partial_{\eta}\right)\right)^{s}\left(-i \bar{y}\left(\partial_{\bar{\xi}}+\partial_{\bar{\eta}}\right)\right)^{s} & \\
& \left.\sum_{p_{1}, q_{1}} \frac{1}{p_{1} ! q_{1} !} C_{\alpha\left(p_{1}\right) \dot{\alpha}\left(q_{1}\right)}(x) \xi^{\alpha\left(p_{1}\right)} \bar{\xi}^{\dot{\alpha}\left(q_{1}\right)} \sum_{p_{2}, q_{2}} \frac{1}{p_{2} ! q_{2} !} C_{\beta\left(p_{2}\right) \dot{\beta}\left(q_{2}\right)} \eta^{\beta\left(p_{2}\right)} \dot{\eta}^{\dot{\beta}\left(q_{2}\right)}\right|_{\eta=0, \xi=0} .
\end{aligned}
$$

It is then easy to perform all required differentiations ending up with

$$
(\beta \gamma)^{s+1} \alpha_{1}^{n} \tau \rightarrow(-1)^{s} \frac{y^{\alpha(s)} \bar{y}^{\dot{\alpha}(s)}}{s !^{2}} \sum_{p, q=0}^{s}(-1)^{q}\left(\begin{array}{l}
s \\
p
\end{array}\right)\left(\begin{array}{l}
s \\
q
\end{array}\right) \frac{(-i)^{n}}{n !} C_{\alpha(s-p) \beta(n) \dot{\alpha}(s-q)} C_{\alpha(p)}^{\beta(n)} \dot{\alpha}(q),
$$

together with its conjugate piece which can be obtained by replacing $\alpha_{1}$ with $\alpha_{2}$. Notice that the spin of a zero form $C_{\alpha(n) \dot{\alpha}(m)}$ is given by $\frac{|n-m|}{2}$ so that the first 0-form has spin $s_{1}=\frac{|n-(p-q)|}{2}$ while the second has spin $s_{2}=\frac{|n+(p-q)|}{2}$. The number of derivatives is instead $s_{1}+s_{2}+\min (s-p+n, s-q)+\min (p+n, q)$. A particularly interesting case is when the canonical current has the maximum number of derivatives $s+s_{1}+s_{2}$. For $s_{1}=s_{2}$ this coupling is realised with $p=q$ and $s_{1}=s_{2}=\frac{n}{2}$. The $s-0-0$ current is a particular case of the latter for $n=0$. In the $s-s_{1}-s_{2}$ case $n=0$ reproduces Bel-Robinson type currents $[23] .^{12}$

\section{B Fixing the 2pt normalisations in Vasiliev's equations holographically}

An important subtlety to correctly interpret coefficients of currents at the equations of motion level is to determine the kinetic term normalisation for the Fronsdal fields. Such normalisation indeed play a key role to fix the cubic couplings in [7]. In the following we will recall all these normalisation fixing the notation. Furthermore we will fix the kinetic-term normalisation for all HS fields by matching holographically the sources coming from the $0-0-s$ cubic couplings.

First of all it is important to fix the convention for spinorial indices as in [3] with

$$
h_{\mu}^{\alpha \dot{\alpha}} h_{\beta \dot{\beta}}^{\mu}=\epsilon_{\beta}{ }^{\alpha} \epsilon_{\dot{\beta}}^{\dot{\alpha}}, \quad h_{\mu}^{\alpha \dot{\alpha}} h_{\alpha \dot{\alpha}}^{v}=\delta_{\mu}^{v},
$$

and in Poincaré coordinates:

$$
h_{\mu}^{\alpha \dot{\alpha}} d x^{\mu}=\frac{1}{2 z} \sigma_{\mu}^{\alpha \dot{\alpha}} d x^{\mu}, \quad h_{\alpha \dot{\alpha}}^{\mu}=z \sigma_{\alpha \dot{\alpha}}^{\mu}, \quad g_{\mu v}=\frac{1}{2 z^{2}} \eta_{\mu v} .
$$

Here $\epsilon^{\alpha \beta}=-\epsilon^{\beta \alpha}, \epsilon^{12}=1$ and $\epsilon=i \sigma_{2}$ with $\sigma_{i}^{\alpha \dot{\alpha}}, i=1,2,3$ being the Pauli matrices. Notice that in this note we work with the choice $\Lambda=2$. Fronsdal equations then read:

$$
\frac{1}{4(s-1)}\left[\square+2\left(s^{2}-2 s-2\right)+\ldots\right] \phi_{\alpha(s) \dot{\alpha}(s)}=J_{\alpha(s) \dot{\alpha}(s)} .
$$

\footnotetext{
${ }^{12}$ In the $n=0$ case the same generating function can also be written in more conventional form as
}

$$
(\beta \gamma)^{s+1} \tau \quad \longrightarrow \quad C(y, \bar{y}) C(-y, \bar{y}) .
$$


where we have included the factor $\frac{1}{4(s-1)}$ coming from solving torsion as described in [3]. The mass term is dressed by a factor of 2 coming from our conventions for $\Lambda$. The mapping between spinorial and vectorial indices is achieved via the vielbein as:

$$
\phi_{\mu(s)} \equiv \phi_{\alpha(s) \dot{\alpha}(s)}(x) \underbrace{h_{\mu}^{\alpha \dot{\alpha}} \cdots h_{\mu}^{\alpha \dot{\alpha}}}_{s},
$$

which follows from (69).

The holographically reconstructed equations can be extracted from the 0-0-s cubic coupling:

$$
\mathcal{L} \sim \sum_{s}\left[\frac{N_{s}^{2}}{2^{s+2}} \phi_{\mu(s)} \square \phi^{\mu(s)}+\ldots\right]-\underbrace{\frac{2^{4}}{\sqrt{N}}}_{g} \sum_{s} N_{s} N_{0}^{2} \frac{2^{-\frac{s}{2}}}{\Gamma(s)} i^{s} \phi^{\mu(s)}\left(\frac{1}{2^{s}} \phi \stackrel{\leftrightarrow}{\nabla}_{\mu(s)} \phi+\ldots\right),
$$

with an arbitrary normalisation $N_{s}$ for the Fronsdal kinetic term. They read: ${ }^{13}$

$$
(\square-4) \phi(x)=g N_{s} \frac{2^{2-\frac{s}{2}} i^{s}}{\Gamma(s)} \phi^{\mu(s)} \nabla_{\mu(s)} \phi=g N_{s} \frac{2^{2-\frac{s}{2}}}{\Gamma(s)} \phi^{\alpha(s) \dot{\alpha}(s)} C_{\alpha(s) \dot{\alpha}(s)},
$$

where we have used that on the scalar sector of the zero form:

$$
\widetilde{D} C=0 \quad \longrightarrow \quad C_{\alpha(s) \dot{\alpha}(s)}(x)=i^{n} \underbrace{\nabla_{\alpha \dot{\alpha}} \cdots \nabla_{\alpha \dot{\alpha}}}_{s} \phi(x) .
$$

The above equations allow to determine holographically the normalisation of the kinetic term used by Vasiliev's equations simply from the $0-0-s$ source to the scalar equation which can be extracted from the standard twisted-adjoint structure constants:

$$
\widetilde{D} C=\omega \star C-C \star \pi(\omega) .
$$

Upon translating the above equations to standard Klein-Gordon equations we arrive to:

$$
(\square-4) \phi(x)=\frac{4}{\Gamma(s)^{2}} \phi^{\alpha(s) \dot{\alpha}(s)} C_{\alpha(s) \dot{\alpha}(s)},
$$

from which comparing with (74) we can determine the normalisation $N_{s}$ used by Vasiliev's equations:

$$
N_{s}=\frac{1}{g} \frac{2^{\frac{s}{2}}}{\Gamma(s)}, \quad s>0 .
$$

In the latter normalisation one then gets the following coupling constants for the source to the Fronsdal equations:

$$
\left[\square+2\left(s^{2}-2 s-2\right)+\ldots\right] \phi_{\mu(s)}(x)=\underbrace{\frac{g N_{0}^{2}}{N_{s}} \frac{2^{\frac{s}{2}+1}}{\Gamma(s)}}_{=2 g^{2} N_{0}^{2}} i^{s}\left(\frac{1}{2^{s}} \phi \stackrel{\leftrightarrow}{\nabla}_{\mu(s)} \phi+\ldots\right),
$$

which, taking into account the normalisation (3) for the canonical current, determines the value for the coefficient $\alpha_{s}$ in (4) up to a spin-independent constant:

$$
\alpha_{s}=2^{1-s} g^{2} N_{0}^{2} .
$$

Similar results can be obtained for all other couplings of [7].

\footnotetext{
${ }^{13}$ Notice that a factor $2^{-s}$ comes from the non-canonically normalised metric in the contractions between HS field and derivatives. A further factor of 2 comes from the non canonical normalisation for the Laplacian and another factor of 2 comes from the variation with respect to the scalar field.
} 


\section{References}

[1] M.A. Vasiliev, Phys. Lett. B243, 378 (1990)

[2] P. Kessel, G. Lucena Gómez, E. Skvortsov, M. Taronna, JHEP 11, 104 (2015), 1505.05887

[3] N. Boulanger, P. Kessel, E.D. Skvortsov, M. Taronna, J. Phys. A49, 095402 (2016), 1508. 04139

[4] N. Boulanger, S. Leclercq, P. Sundell, JHEP 08, 056 (2008), 0805 . 2764

[5] E.D. Skvortsov, M. Taronna, JHEP 11, 044 (2015), 1508 . 04764

[6] M. Taronna, Pseudo-local Theories: A Functional Class Proposal, in International Workshop on Higher Spin Gauge Theories Singapore, Singapore, November 4-6, 2015 (2016), 1602 .08566, https://inspirehep.net/record/1424813/files/arXiv: 1602.08566.pdf

[7] C. Sleight, M. Taronna, Phys. Rev. Lett. 116, 181602 (2016), 1603.00022

[8] M. Taronna, Master Thesis (2010), 1005.3061

[9] A. Sagnotti, M. Taronna, Nucl. Phys. B842, 299 (2011), 1006.5242

[10] S.F. Prokushkin, M.A. Vasiliev, Theor. Math. Phys. 123, 415 (2000), [Teor. Mat. Fiz.123,3(2000)], hep-th/9907020

[11] M.A. Vasiliev (2016), 1605.02662

[12] M.A. Vasiliev, JHEP 06, 031 (2015), 1502.02271

[13] X. Bekaert, J. Erdmenger, D. Ponomarev, C. Sleight, JHEP 11, 149 (2015), 1508 . 04292

[14] R.R. Metsaev, Mod. Phys. Lett. A6, 2411 (1991)

[15] R.R. Metsaev, Mod. Phys. Lett. A6, 359 (1991)

[16] X. Bekaert, J. Erdmenger, D. Ponomarev, C. Sleight, JHEP 1503, 170 (2015), 1412 .0016

[17] X. Bekaert, J. Erdmenger, D. Ponomarev, C. Sleight, Bulk quartic vertices from boundary four-point correlators, in International Workshop on Higher Spin Gauge Theories Singapore, Singapore, November 4-6, 2015 (2016), 1602.08570, http://inspirehep.net/record/1424869/files/arXiv: 1602.08570.pdf

[18] F. Kristiansson, P. Rajan, JHEP 04, 009 (2003), hep-th/0303202

[19] J. Maldacena, A. Zhiboedov, Class. Quant. Grav. 30, 104003 (2013), 1204 . 3882

[20] M. Taronna, Ph.D. thesis, Pisa, Scuola Normale Superiore (2012), 1209.5755, https://inspirehep.net/record/1188191/files/arXiv:1209.5755.pdf

[21] M.A. Vasiliev, Nucl. Phys. B324, 503 (1989)

[22] M.A. Vasiliev, Phys. Lett. B238, 305 (1990)

[23] O.A. Gelfond, E.D. Skvortsov, M.A. Vasiliev, Theor. Math. Phys. 154, 294 (2008), hep-th/0601106 